\title{
TRANSCODIFICACIÓN VERSUS COMUNICACIÓN - CONSIDERACIONES METODOLÓGICAS ACERCA DE LA ENSEÑANZA DE LA INTERPRETACIÓN SIMULTÁNEA ALEMÁN- ESPAÑOL
}

\author{
Christoph Ehlers \\ Universidad de Sevilla
}

\begin{abstract}
This paper reflects the author's experience of teaching a 50-hour preliminary course of simultaneous translation (German-Spanish). Simultaneous on-line-processing of input in one code and output in another, the so-called 'split attention', is most likely to furbish interference in word-to-word translation. The first step to overcome these hurdles is to gain consciousness of the role of the interpreter as a mediator between persons and cultures instead of someone who pours the word from one linguistic code into another. In order to achieve distance from the text and see it as information to be carried, trainees have to learn to rely heavily on top-down-processing by focusing on content. Second and most conspicuously, on-line oral simultaneous reception and production has to be tackled by specific training activities, such as 'salami-technique', 'split attention', 'lagging' and 'leading'. These techniques of foreshadowing and retaining information are specifically important in a language contact with German involved, which typically poses heads at the end of the verbal or nominal phrase.
\end{abstract}

\section{INTRODUCCIÓN}

En primavera del 2001, el autor fue encargado de diseñar e impartir un curso-módulo de 'Iniciación a la Interpretación Simultánea Alemán-Español' de sólo cincuenta horas de duración ${ }^{1}$, concentradas en cuatro semanas con dos horas diarias de clase. De ninguna manera se consideraba que en cuatro semanas se pudiera conseguir lo que las Facultades de Traducción e Interpretación consiguen en cuatro años y que los estudiantes del módulo pudieran entrar sin más en cabina. Quiero destacar, desde el principio, el caracter meramente introductorio del módulo, englobado en un máster de orientación decididamente práctica.

En este artículo quisiera presentar una especie de memoria crítica de las actividades realizadas, mis reflexiones durante la elaboración de la programación y su resultado final,

\footnotetext{
${ }^{1}$ En el marco del programa "MATRI - Máster Universitario de Traducción de Textos con Fines Específicos, Interpretación y Doblaje de las Lenguas Española y Alemana" de la Universidad de Sevilla. Doy las gracias a Rafael López-Campos Bodineau, coordinador del mismo, por haberme invitado a colaborar.
} 
deteniéndome también en aquellos aspectos que afectan a mis dos campos de actividad: enseñanza de segundas lenguas (EL) e interpretación simultánea (IS).

\section{LOS APRENDICES. CONOCIMIENTOS Y EXPECTATIVAS}

Los datos personales de los aprendices ${ }^{2}$ pertinentes para nuestra actividad fueron elicitados en una primera unidad de toma de contacto, centrada en las experiencias previas en la traducción e interpretación (T/IS) y las expectativas de cada aprendiz para con este curso. Los aprendices, fotografiados in situ con Polaroid, entrevistaban a un compañero y lo presentaban a la clase en un panel en la pared, todo en alemán. Las preguntas incluían: procedencia, contactos con la lengua / cultura alemana, expectativas para el curso, planes profesionales, y, finalmente, un dibujo representando una metáfora de la IS. Este procedimiento, probado en seminarios y clases de lengua, perseguía varios fines: crear lo antes posible un sentimiento de grupo, necesario en un curso con prolongado contacto diario; involucrar al aprendiz mediante su retrato visible durante todo el curso; verbalizar las expectativas para poder contrastarlas al final del curso con los resultados obtenidos; conceptualizar en una metáfora la visión propia de la IS, también para ver al final del curso si se han producido cambios positivos o negativos en esta percepción.

Esta primera encuesta permitió descubrir que los catorce aprendices matriculados presentaban una competencia lingüística muy heterogénea. Dado que se debía traducir a la lengua A (materna), y que sólo se trataba de un curso de iniciación o primera aproximación, se admitían interesados con conocimientos del alemán aun muy lejos de ser suficientes para la práctica profesional. Sólo dos alumnas tenían experiencia profesional previa como intérprete de juzgados y consecutiva bilateral, respectivamente. Las expectativas eran en su gran mayoría modestas, realistas a la vista del nivel idiomático. Sólo cuatro aprendices indicaban objetivos profesionales directamente relacionados con la T/IS (Falta la aclaración de T). Ello no impidió que la motivación fuera grande: todos querían aprender y experimentar, al menos en situación de ensayo, la sensación de ser un 'acróbata lingüístico'. Para mantener esta disposición positiva, se subrayaron desde el principio dos aspectos: en primer lugar, el papel del intérprete como mediador entre personas y culturas, no como transcodificador entre sistemas lingüísticos, y segundo, la visión de la IS como una especie de deporte mental, una destreza que sólo se puede adquirir mediante la práctica.

\section{LOS OBJETIVOS}

Los objetivos se pueden plantear desde dos puntos de vista: uno más general, válido para la IS en cualquier contexto de trabajo, y otro, más específico, para preparar al aprendiz para el entorno laboral concreto que le esperaría, Andalucía Occidental en nuestro caso. Para esto último, se incluyó en la parte introductoria un epígrafe sobre el contexto laboral,

\footnotetext{
${ }^{2}$ Uso deliberadamente el término 'aprendiz' para subrayar que estamos hablando de una destreza práctica, como las propias de un oficio. Por otra parte, el término tiene amplia aceptación en la literatura ELE, trasiego del inglés 'learner'.
} 
el mercado local "libre", con indicaciones sobre asociaciones, estrategias para obtener trabajos, condiciones contractuales etc. (véase también. Abril, Collados y Martín 2000). Pero, por supuesto, los buenos intérpretes poseen una serie de habilidades idénticas en todo el mundo que son las que se pretendían transmitir en este curso.

Para poder suministrar un producto final satisfactorio - cuyas características principales son la reproducción fiel del contenido y la claridad - el intérprete requiere, aparte naturalmente de su competencia lingüística y su talento, una serie de técnicas específicas que abarcan desde el trabajo terminológico preparatorio, de fundamental importancia, hasta las técnicas de 'gestión de crisis' en cabina. Entre estos pilares se insertan las siguientes 'subdestrezas' (véase Kurz 1995): fluidez, cohesión lógica, comportamiento profesional, dominio terminológico, conocimiento enciclopédico (Thesaurus), capacidad de concentración, competencia gramatical, competencia intercultural, modulación de la voz y capacidad de decisión. Estos factores fueron evaluados y jerarquizados por cada participante, y después discutidos en el pleno. De esta manera, los propios aprendices reflexionaban sobre las destrezas concretas que querían adquirir, aumentando así su implicación en los objetivos y contenidos del curso. En ELE, este tipo de actividad, tratado en el contexto de las estrategias metacognitivas, en los últimos años ha obtenido cada vez más protagonismo (véase Wenden 1991). Se trata de convertir al aprendiz en sujeto de su propio proceso de aprendizaje, haciéndole reflexionar sobre sus metas. El resultado de esta negociación, ciertamente dirigida, fue el temario de contenidos previamente elaborado por mí (véase Anexo).

\section{LOS CONTENIDOS. LA BASE COGNITIVA}

Estos aspectos sirvieron, pues, para ordenar los contenidos teóricos. Acompañados de sus respectivos ejercicios prácticos se sucedían de la siguiente manera: competencia lingüística, IS como acto de comunicación complejo, fidelidad al original y reproducción del sentido, conocimiento enciclopédico y preparación terminológica, atención distribuida y anticipación.

La introducción teórica ocupó la cuarta parte del horario total, si bien siempre entreverado con ejercicios prácticos como traducción 'a la vista', reformulación de textos e imágenes en español y alemán, análisis comunicativo de situaciones de trabajo reales (las "preguntas- $\iota \mathrm{Q}$ ?" - qué a quién para qué cuándo dónde etc.), conversión de hipotaxis en parataxis ('técnica salami' de Jones 1998) etcétera. (véase anexo). Así se presentaron las bases de la actuación del IS desde los puntos de vista de la teoría de la comunicación (Pöchhacker 1994), lingüística textual (Nord 1988) y psicología cognitiva (Kurz 1996). Centramos nuestra atención en que la IS es un servicio con una función comunicativa en la que se transmiten ideas y no palabras, o sea, que el intérprete no es un transcodificador sino un comunicador inmerso en una compleja 'constelación textual' (Nord 1988) con sus múltiples condicionantes funcionales, económicos y personales.

Los objetivos prácticos son evidentes: La IS constituye un complejísimo proceso de procesamiento de información que comprende una serie de subdestrezas interdependientes. "La única actividad que desmiente el axioma según el cual el cerebro no puede realizar dos 
tareas complejas a la vez" (Viaggio 1988). Este proceso tan exigente puede aprenderse -y enseñarse- mucho mejor si se descompone la destreza global 'interpretación simultánea' en varias destrezas parciales que se van enseñando y entrenando progresivamente, de menor a mayor dificultad. (véase Kurz 1995). Las subdestrezas se definieron de la siguiente manera:

1. Memoria a corto y largo plazo; acceso léxico.

2. Capacidad de escuchar y hablar a la vez, la así llamada 'split attention' o atención distribuida.

3. Estrategias como la anticipación ('leading'), de gran importancia con una lengua de partida de núcleo final como el alemán, y el retraso ('lagging') para dar tiempo a la comprensión y el análisis.

\subsection{Memoria a corto y largo plazo. Acceso léxico}

Como el maestro de ajedrez, que reconoce hasta 5000 situaciones de juego diferentes comprimiéndolas en 'chunks' o unidades de memoria, también el intérprete tiene que ir construyéndose una red de patrones asociativos que regulan su procesamiento. (Igoa y García Albea 1988). El reconocimiento y la activación de estos patrones puede acelerarse mediante el ejercicio. El proceso es más rápido cuanto más información puede procesarse en forma de 'chunks' (el número mágico son de manera aproximada 5 simultáneamente), lo cual significa que el intérprete tiene que familiarizarse con el mayor número posible de patrones mentales y verbales. En el curso, este objetivo se persiguió, en la parte teórica, mediante la delimitación de las áreas temáticas a comercio, política regional y conferencias, y en la práctica, con el trabajo realizado en el laboratorio de idiomas, con muchas fases de repetición y autoescucha.

En cuanto a la competencia léxica, resultó muy útil el 'modelo gravitacional de accesibilidad léxica' de Gile (1994) que explica de modo gráfico la constitución y, sobre todo, conservación de un amplio repertorio léxico y expresivo en ambas lenguas. En él, la competencia individual se contempla como un sistema concéntrico alrededor de un núcleo, el cual contiene las reglas secuenciales y unidades léxicas siempre disponibles para la producción, y una periferia con los ítems sólo accesibles para la comprensión. La ventaja didáctica del modelo consiste en su carácter dinámico: cuanto más se activa una unidad o regla determinada mediante estimulación, más cerca se encuentra del núcleo, 'arrastrando' además otras unidades cognitivamente asociadas, y cuanto menos activadas, más cerca se sitúa de la periferia.

Fue en la comprensión donde los aprendices, casi todos hablantes maternos del español, mostraron sus limitaciones más serias por su falta de automatización receptiva, y donde los dos aprendices bilingües empezaron a mostrar claras ventajas. Es decir, se apoya la observación defendida por la escuela rusa de IS que la interpretación no necesariamente debe ser hacia la lengua materna sino, por la ventaja arriba señalada, desde ella hacia la lengua B: la descodificación requiere menos energía de procesamiento que se puede dedicar a la producción. 


\section{2 'Split attention'}

También resultó muy útil, por sencillo y gráfico, el 'effort-model' de Gile (1995a) que destaca el delicado equilibrio entre escucha, análisis y reformulación en el proceso de la IS. $\mathrm{La}$ idea básica consiste en que las operaciones mentales no-automatizadas requieren mucho más capacidad de procesamiento, que es limitada, que las automatizadas. La IS es una actividad no-automatizada (estímulos débiles y/o desconocidos, necesidad de almacenamiento a corto plazo, planificar la reacción espontánea etc.) que, como dijimos, puede automatizarse mediante la práctica y además, se ve facilitada por el procesamiento 'top-down' de la información, es decir, guiada por claves pragmáticas como contexto, suposiciones y el conocimiento no específicamente lingüístico del intérprete. Cuanto más procesamiento a través del contexto ('top-down'), cuantos más 'chunks' y rutinas creadas, tanto menos capacidad de procesamiento se requiere. Esta capacidad global se ha de repartir de la manera más equilibrada posible entre la energía empleada para la recepción, para la reformulación y para la memoria ${ }^{3}$.

$\mathrm{La}$ atención distribuida fue practicada en el laboratorio con varios tipos de ejercicios: 'shadowing' (repetición con ligero retraso, o una variante muy interesante y entretenida propuesta por Kurz (Dollerup et al. 1994), consistente en responder a preguntas sencillas mientras se está escuchando la siguiente pregunta), retraso ('lagging') y anticipación ('leading')

\subsection{Estrategias}

La anticipación ('leading') constituye una subdestreza básica del intérprete para planificar su discurso y descargar el esfuerzo de procesamiento. Aquí confía en el procesamiento 'top-down' que le permite anticipar por contexto o co-texto, conocimiento lingüístico y extralingüístico. Es especialmente relevante si la lengua de partida, en este caso el alemán, es de tipo núcleo-final: el núcleo predicativo del sintagma ha de ser 'adivinado' en base a los modificadores o argumentos que le preceden. Esta dificultad fue ensayada primero con textos escritos del que habían sido tachadas palabras inducibles por contexto, verbos al final de oraciones subordinadas, participios verbales etc. En un siguiente paso, el profesor leía un texto en voz alta con 'pausas de carraspeo' en los que los participantes formulan sus hipótesis sobre la continuación del discurso.

\section{EL TRABAJO EN EL LABORATORIO DE IDIOMAS}

El conocimiento fáctico, adquirido en la parte teórica, debía convertirse en conocimiento procedural, una habilidad no consciente adquirida por mecanización. Los objetivos del trabajo con micrófono y auriculares eran los siguientes: primero, crear el hábito de hablar y escuchar a la vez ('split attention'), segundo, encontrar la distancia

\footnotetext{
${ }^{3}$ Gile lo ilustra con la metáfora del tabernero que ha de cuidar que esté lleno el barril de vino (capacidad global) y las copas de sus tres clientes (recepción, reformulación y memoria).
} 
temporal ideal entre texto de entrada y salida ('lagging'), estipulado por Seleskovitch y Lederer (1989) en dos 'unidades de sentido', tercero, estimular la memoria a corto plazo, y cuarto, liberación del corset del texto de salida. Este último punto fue subrayado como una ventaja frente a la traducción escrita, considerada generalmente como más 'fácil' pero en realidad más exigente en cuanto a fidelidad literal al original.

Antes de comenzar se expusieron las 'cuatro reglas de oro del intérprete' (Jones 1998) que debían acercar a la situación real: nunca hacer comentarios o ruidos con el micrófono abierto, no repetirse en busca de sinónimos o una expresión más acertada, no emitir expresiones de duda como 'mmm' y, por último, siempre terminar las frases, no emitir anacolutos.

En esta fase, cada ejercicio fue repetido hasta que los aprendices eran capaces de realizarlo sin error, lo cual aumentó su confianza:

1. No se empezó con frases sino con palabras, en un ejercicio de 'lagging' (retraso): listas de palabras relacionadas semánticamente (p. ej. campo léxico de 'la casa') que debían traducirse con un retraso que con cada repetición iba en aumento. Al principio, se traducía con dos palabras de retraso, al final con cuatro

2. En vez de traducir palabras, en el siguiente ejercicio, adaptado de Kurz (1994) se respondía en español a unas 30 preguntas absolutas (sí/no), hechas en alemán, del tipo ‘¿Es París la capital de Bulgaria?' mientras ya se escuchaba la siguiente pregunta. Había que responder con oraciones asertivas completas.

3. Las preguntas ya no eran absolutas, sino relativas: ‘Cuál es la montaña más alta de la Península'? etc

En el siguiente bloque ya se trabajaba con textos completos, entrevistas poco técnicas, de un lenguaje estándar común, inicialmente destinadas a ejercicios de comprensión oral en la clase de alemán. Tras varios ensayos se consolidó una secuencia práctica que resultó provechosa:

1 Los aprendices escogieron una entrevista de una lista de 30, guiados por el interés personal en su contenido, p. ej. 'Entrevista a un ciego'

2 En una fase de 'sensibilización', término proveniente de la enseñanza comunicativa de idiomas, se produce una conversación en el pleno acerca del tema con experiencias personales o conocimientos previos. El profesor va moderando y apuntando los términos relacionados con el tema, p.e.'perro-guía' con su traducción al alemán. De este modo, se crea una red léxica o glosario que facilita la comprensión del texto

3 Con esta lista de términos se realiza un primer pase de ejercicios de 'lagging' (retraso), arriba descritos

4 Se reparte la trascripción de la entrevista que en parejas de a dos se traduce 'a la vista', con anotaciones de términos específicos o difíciles 
5 Uno tras otro, los aprendices traducen de nuevo a la vista, pero en el pleno. Se comentan dificultades y se recogen nuevos términos para la lista de 'lagging' en la pizarra

6 Otro pase de 'lagging' con la lista ampliada y referida el texto en cuestión

7 Primer pase lento del texto, sin trascripción delante, con pausas tras cada 2-3 unidades de sentido

8 Se repite el texto $\sin$ pausas

9 Los aprendices escuchan sus versiones y comentan sus observaciones en el pleno

10 Se repite una última vez

Durante la interpretación, el profesor puede escuchar el trabajo de los aprendices, comentar 'on line' o tomar notas para comentar después en el pleno.

Después de 'entrenar' de este modo con cinco textos previamente trabajados con traducción a la vista, se pasó al vídeo sin trascripción. Se usaron grabaciones de discursos de políticos de gobierno y oposición ${ }^{4}$ con temática dispersa pero de actualidad política (fiebre aftosa, política sanitaria etc.). El vídeo significa un paso más hacia la situación real en cabina: el orador se dirige a un público amplio pero concreto y usa recursos retóricos y paralingüísticos como la entonación, expresión facial y gesticulación que obligan al intérprete a actuar/mimetizar el discurso. La secuenciación didáctica se orientaba en la anterior:

1 Conversación acerca de los temas contenidos en los discursos y comentados por el profesor. 'Brainstorming' y red léxica

2 Ejercicio de 'lagging' con este vocabulario

3 Interpretación con pausa

4 Interpretación sin pausa

De esta manera se fue creando una rutina de secuencias de trabajo que daba seguridad a los aprendices. La relación con técnicas EL2 se limitaba a la selección por los propios aprendices de los temas tratados en cinta audio (autodeterminación de contenidos), el trabajo preparatorio en parejas y la elaboración propia de los glosarios.

\section{CONCLUSIÓN}

En muchos sentidos, me resultó útil mi experiencia práctica tanto en la IS como en la EL2. No sólo los aprendices aprenden haciendo ('learning by doing'), sino también el profesor aprende enseñando. Tanto más en cuanto fue la primera vez que me enfrentaba a un objetivo tan complejo como la(s) destreza(s) básica(s) de la IS: actuación comunicativa adecuada, reformulación fiel al sentido del texto original y claridad de exposición, metas alcanzables mediante las subdestrezas de atención distribuida ('split attention'), retraso ('lagging') y anticipación ('leading').

\footnotetext{
${ }^{4}$ Sacados de la excelente cadena monográfica de televisión 'Phoenix'.
} 


\section{REFERENCIAS BIBLIOGRÁFICAS}

DANKS, J.H. / SHREVE, G.M. / FOUNTAIN, S.B. / MCBEATH, M.K. (eds.), Cognitive Processes in Translation and Interpreting, Thousand Oaks/London/New Delhi, Sage Publications, 1997.

DOLLERUP, C. / LINDEGAARD, L. (eds.), Teaching Translation and Interpreting. Insights, Aims, Visions, 3 tomos, Amsterdam, Benjamins, 1994.

GILE, D., Basic Concepts and Models for Interpreter and Translator Training, Amsterdam Benjamins, 1995.

GRAN, L. / DODDS, J., The Theoretical and Practical Aspects of Teaching Conference Interpretation, Udine, Campanotto, 1989.

IGOA, J.M / GARCÍA ALBEA, J.E, "Procesamiento en la comprensión y la producción de oraciones en una tarea de traducción oral simultánea”, Cognitiva 1-2 (1988), 123153.

JONES, R., Conference Interpreting Explained, Manchester, St. Jerome, 1998.

KURZ, I., Simultandolmetschen als Gegenstand der interdisziplinären Forschung, Wien, WUV Wiener Universitätsverlag, 1996.

PADILLA BENÍTEZ, P., "Procesos de memoria y atención: Hacia una teoría cognitiva de la interpretación”, Sendebar Vol.5 (1994), pp. 55-66.

SCHMIDJELL, A., Lernen ist lernbar. Autonomes Lernen und Lernstrategien, Munich, Hueber, 2001.

SELESKOVITCH, D. / LEDERER, M., Pédagogie raisonnée de l'ínterprétation, Paris, Didier, 1989.

SELESKOVITCH, D. / LEDERER M., A systematic approach to teaching interpretation, Silver Spring MD, Registry of Interpreters for the Deaf, 1995.

SNELL HORNBY, M. et al. (eds.), Handbuch Translation, Tübingen,.Stauffenberg, 1998. WENDEN, A., Learner Strategies for Learner Autonomy, New York, Prentice Hall, 1991. 
ANEXO: Esquema de planificación del curso

\begin{tabular}{|c|c|}
\hline CONTENIDOS & EJERCICIOS PRÁCTICOS \\
\hline I. Introducción & $\begin{array}{l}\text { 0. Entrevistas mutuas, explicitación de expectativas, metas. } \\
\text { Fotos, poster. }\end{array}$ \\
\hline \multicolumn{2}{|l|}{ 1. Modalidades y términos profesionales } \\
\hline \multicolumn{2}{|l|}{ 2. Contexto laboral } \\
\hline \multicolumn{2}{|l|}{ 3. Historia de la IS } \\
\hline $\begin{array}{l}\text { II. Técnicas de IS - ¿Qué es lo que debe } \\
\text { saber hacer un intérprete? }\end{array}$ & 1. Jerarquización de las 'destrezas del intérprete' \\
\hline \multicolumn{2}{|l|}{ 1. Concentración } \\
\hline 2. Competencia lingüística & 2. Reformulación 'a la vista' de noticias del periódico \\
\hline 3. Comunicación & 3. Resumen de noticias del periódico \\
\hline 3.1. IS como acto de comunicación & 4. Análisis de encargos reales mediante "preguntas Qu- \\
\hline \multicolumn{2}{|c|}{$\begin{array}{l}\text { 3.2. Los componentes de la comunicación } \\
\text { en la IS }\end{array}$} \\
\hline \multicolumn{2}{|l|}{ 3.3. El concepto de 'calidad' en IS } \\
\hline 4. Fidelidad y reproducción del sentido & 5. Reformulación de información visual \\
\hline 4.1. Principios de fidelidad textual & 6. 'Técnica del salami' con textos escritos \\
\hline 5. Comprensión y conocimiento fáctico & $\begin{array}{l}\text { 7. Evaluación del grado de dificultad de distintos textos } \\
\text { técnicos } \\
8 \text {. Ejercicios de sinonimia y hiperonimia. Pirámides } \\
\text { conceptuales }\end{array}$ \\
\hline \multicolumn{2}{|l|}{ 5.1. Conocimiento fáctico } \\
\hline \multicolumn{2}{|l|}{ 5.2. Estrategias de aprendizaje fáctíco } \\
\hline \multicolumn{2}{|l|}{ 5.3. Fuentes de documentación } \\
\hline $\begin{array}{l}\text { 6. Del oido a los labios - El 'modelo } \\
\text { secuencial' de Gile }\end{array}$ & $\begin{array}{l}\text { Laboratorio de Idiomas } \\
\text { 9. 'Shadowing': traducción de listas de palabras } \\
\text { retraso }\end{array}$ \\
\hline 7. IS y esfuerzo de procesamiento & $\begin{array}{l}\text { 10. 'Lagging': responder a } \\
\text { a. preguntas totales (síno) } \\
\text { b. preguntas parciales (Qu-) }\end{array}$ \\
\hline 7.1. El 'Effort-Model' de Gile & \multirow{6}{*}{$\begin{array}{l}\text { 11. Interpretación de entrevistas audio } \\
\text { a. Brainstorming, sensibilización } \\
\text { b. Traducción a la vista de transcipción } \\
\text { c. Interpretación con pausas } \\
\text { d. Autoescucha y nueva interpretación }\end{array}$} \\
\hline 7.2. Problemas en la IS & \\
\hline 7.3. Anticipación & \\
\hline 7.4. 'Gestión de crisis' en la IS & \\
\hline $\begin{array}{l}\text { 7.4.1. La elección de la estrategia } \\
\text { apropiada }\end{array}$ & \\
\hline 8. Aspectos contrastivos & \\
\hline
\end{tabular}

